\section{The great imitator, revisited}

\section{Nikhil Oliveira, Lorraine Joseph Kandathil, Georgi Tchernev}

Onkoderma - Clinic for Dermatology, Venereology and Dermatologic Surgery, Sofia, Bulgaria

\begin{abstract}
Our study shows an atypical presentation of primary syphilis inappropriately treated with surgery: a 36-year-old male visited our clinic for a consultation 2 weeks after surgery, since he had noticed a recurrent lesion on the glans head of the penis Clinical data, histologic pictures and serological tests, confirmed the diagnosis of an atypical presentation of primary syphilis; in addition, Chlamydia trachomatis infection was found. The patient was started on a course of doxycycline $100 \mathrm{mg}$ tablets, twice daily for 4 weeks. At follow up, there was complete remission.
\end{abstract}

\section{Case Report}

Here we present a case of a 36-year-old male who attended our clinic with the development of a recurrent paraurethral lesion on the head of the glans penis. Prior to his visitation to us, he presented to a urologist with discomfort in the genital area. On examination, a wart-like lesion was identified and surgically excised without postoperative histopathological confirmation. Two weeks after his surgery, the patient noticed a recurrent lesion in the same region and visited our clinic for a consultation. He was apyrexic with no previous urethral discharge or dysuria. He reported no other symptoms and was otherwise healthy. On sexual history, he is active with his current girlfriend and reports no use of condoms. Both him and his partner were negative for HIV and both past sexual histories, when questioned, were unremarkable with no prior sexual partners in the past 10 years. The patient was also noted to be uncircumcised. On physical examination, a single infiltrating paraurethral tumour-like lesion measuring $1.0 \times 0.5 \mathrm{~cm}$ was detected. The lesion contained a glossy surface with an erythematous urethral meatus with a penetrative ulceration communicating with the distal part of the urethra (Figure 1a). There were no bilaterally-enlarged inguinal lymph nodes. No other systemic or oropharyngeal disorders were noted. Based on the clinical data, erythroplasia queyrat was suspected, as well as other possible differentials including spinocellular penile carcinoma, Bowen's diseases or a possible primary syphilis. A core biopsy was taken for further histopathological verification (Figure 1b) which revealed a massive mixed inflammatory infiltrate composed of predominantly lymphocytes and plasmas cells (Figure 1e). There was no evidence of acanthosis with dyskeratotic cells and no mitosis or cellular atypia which gave no further reason to think it was of a malignant origin. Based on the overall histological picture there was a high suspicion of primary syphilitic effect (Figure 1e-f). Further confirmation was necessary with serology which confirmed the diagnosis as an atypical presentation of primary syphilis. Serology showed positive Treponema pallidum hemagglutination assay (TPHA) 1:640 and positive Venereal Disease Research Laboratory (VDRL). In addition to syphilitic positive antibodies our patient had a high IgG endpoint titters of $>$ 1:64 for Chlamydia trachomatis.

Laboratory workup including $\mathrm{CBC}$, liver enzymes, glucose, total cholesterol, urinalysis were normal. Serological testing including HIV was seronegative. Imaging modalities including chest $\mathrm{x}$-ray, abdominal and lymph node echography revealed no pathological findings. The patient was started on a course of doxycycline $100 \mathrm{mg}$ tablets, twice daily for 4 weeks. Patient was advised to follow up after 4 weeks. On follow up, there was complete remission of the infection, including the ulcerative lesion (Figure 1d) with post-therapeutic serology for syphilis and chlamydia being negative.

\section{Discussion and conclusions}

As per the current treatment guidelines, a single dose of benzathine penicillin 2.4 million units intramuscularly is strongly recommended as the standard approach to treating early or latent syphilis. ${ }^{1}$ In a scenario where this treatment regimen cannot be used, i.e. due to penicillin allergy or with stock unavailability, then doxycycline 100 mg orally twice daily for two weeks is suggested. ${ }^{1}$ Although these recommendations are routinely used in clinical practice, very little evidence is available for the outcomes of these therapies in successfully treating early syphilis. ${ }^{1}$ More retrospective data is now emerging that suggest the use of both benzathine penicillin and doxycycline show some success in treating luetic infections. ${ }^{2}$

Due to the lack of availability of benzathine penicillin i.m. in Bulgaria and due to the superimposed infection with chlamydia,
Correspondence: Nikhil Oliveira, Onkoderma - Clinic for Dermatology, Venereology and Dermatologic Surgery, General Skobelev 26, 1606 Sofia, Bulgaria.

Tel.: +359876838005

E-mail: nikhil.oliveira@hotmail.com

Key words: Great imitator, primary syphilis; Chlamydia trachomatis.

Contributions: The authors contributed equally.

Conflict of interest: The authors declare no potential conflict of interest.

Funding: None.

Ethical approval and consent to participate: All procedures performed were in accordance with the ethical standards of the institutional and/or national research committee(s) and with the Helsinki Declaration (as revised in 2013). Written informed consent was obtained for publication of report and accompanying images. A copy of the written consent is available on reasonable request.

Availability of data and material: Data and materials are available by the authors.

Please cite this article as: Oliveira $N$, Kandathil LJ, Tchernev G. The great imitator, revisited. Dermatol Rep 2022;14:9363.

Received for publication: 25 August 2021 Accepted for publication: 4 September 2021.

This work is licensed under a Creative Commons Attribution-NonCommercial 4.0 International License (CC BY-NC 4.0).

Copyright: the Author(s), 2022

Licensee PAGEPress, Italy

Dermatology Reports 2022; 14:9363

doi:10.4081/dr:2022.9363

Publisher's note: All claims expressed in this article are solely those of the authors and do not necessarily represent those of their affiliated organizations, or those of the publisher the editors and the reviewers. Any product that may be evaluated in this article or claim that may be made by its manufacturer is not guaranteed or endorsed by the publisher.

we decided to treat our patient with doxycycline $100 \mathrm{mg}$ twice daily oral tablet regimen of 4 weeks. This clinical decision was based on the concurrent infections as well as the discrepancies in his past history of sexual partners. He reported a single sexual partner, who was negative for syphilis when tested. Our study showed an atypical presentation of primary syphilis that was inappropriately treated with surgery. A recurrent lesion led to further investigation by our 

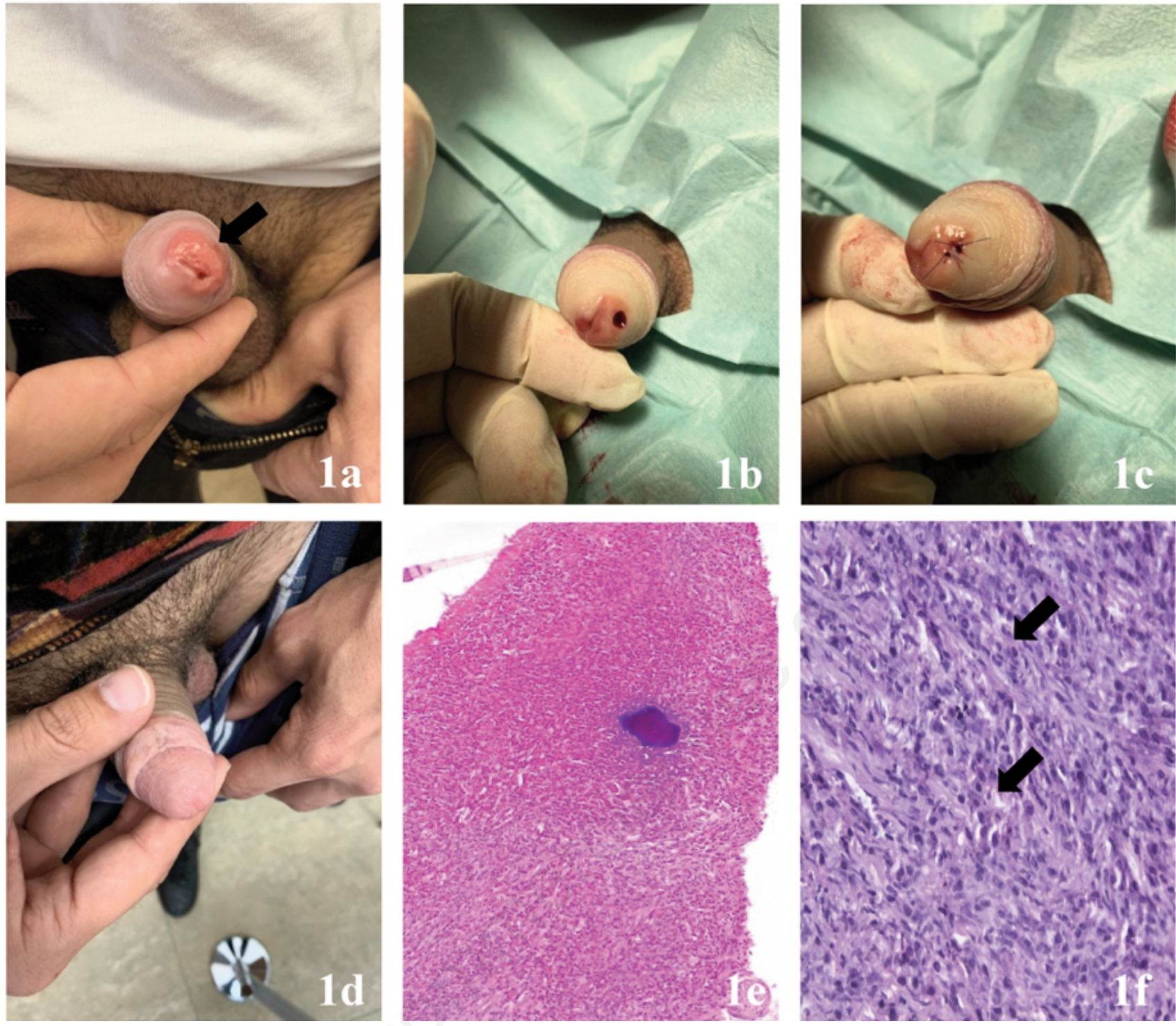

Figure 1. a) Paraurethral deep ulcerating lesion measuring $1.0 \times 0.5 \mathrm{~cm}$. Contains a wart-like shiny surface with raised borders and well demarcated from healthy tissue. b) Core biopsy of the lesion. c) Post-biopsy closure of the defect using single interrupted sutures. d) Clinical picture after a 4 week course of doxycycline $100 \mathrm{mg}$ orally twice daily. Clear remission of the lesion with progressive healing. e) H\&E stained tissue of the lesion on the glans penis. Diffuse inflammatory reaction with predominantly lymphocytes and plasmacytes present ( $\times 40$ magnification). f) H\&E stained tissue of the lesion on the glans penis. Specialised chronic inflammatory cells including lymphocytes and plasmacytes (arrows) seen. No dysplastic or malignant cells were identified ( $\times 100$ magnification).

team of dermatologists that recruited histological analysis to rule out other diseases. Syphilis is referred to as "the great imitator" and can be mistaken for other sexually transmitted conditions or malignancies. ${ }^{3}$ Therefore, it is imperative to suspect a syphilitic infection with any new oral or anogenital lesion and confirm with serological testing. ${ }^{3,4}$ Histology can also be a useful tool to assess both syphilitic infection as well as rule out possible malignancy. ${ }^{4}$ This case presents rare and interesting features of a immunocompetent male that had concurrent primary syphilitic and Chlamydia tra- chomatis infections. He presented with an atypical warty lesion that was surgically excised and reappeared two weeks later as a deep ulcerating lesion. These infections along with the primary lesion was treated with a 4-week dose of doxycycline resulting in complete remission and restitution ad integrum.

\section{References}

1. World Health Organisation. WHO
Guidelines for the Treatment of Treponema pallidum (Syphilis). WHO Press, World Health Organisation

2. Dai T, Qu R, Liu J, et al. Efficacy of Doxycycline in the Treatment of Syphilis. Antimicrob Agents Chemother 2017;61:e01092-16

3. Klausner J. The Great Imitator Revealed: Syphilis. Top Antivir Med 2019;27:71-4.

4. Rockwood N, Nwokolo N. Syphilis the great pretender: when is cancer not cancer? BMJ: Sexual Transmit Infect 2018;94. 